\title{
Multiple-ASSR Interactions in Adults with Sensorineural Hearing Loss
}

\author{
Ieda M. Ishida and David R. Stapells \\ School of Audiology \& Speech Sciences, The University of British Columbia, Friedman Building, Room 443, 2177 Wesbrook Mall, \\ Vancouver, BC, Canada V6T $1 Z 3$ \\ Correspondence should be addressed to David R. Stapells, stapells@audiospeech.ubc.ca
}

Received 19 June 2012; Accepted 26 July 2012

Academic Editor: Leonard P. Rybak

Copyright ( 2012 I. M. Ishida and D. R. Stapells. This is an open access article distributed under the Creative Commons Attribution License, which permits unrestricted use, distribution, and reproduction in any medium, provided the original work is properly cited.

\begin{abstract}
The multiple auditory steady-state response (multiple-ASSR) technique, where thresholds for up to 8 frequencies (4 in each ear) are obtained simultaneously, is currently of great interest for audiometric assessment of infants. Although threshold estimates using the multiple-ASSR appear to be reasonably accurate, it is not currently known whether it is more efficient to use multiple stimuli or single stimuli when testing individuals with sensorineural hearing loss (SNHL). The current study investigated the effect of single versus multiple simultaneous stimuli on the 80 - and 40-Hz ASSRs in adults with normal hearing or SNHL. Results showed significant interactions (i.e., decreased amplitudes) for both ASSRs going from single to multiple stimuli in one ear. Going from multiple one ear to multiple two ears did not further reduce the amplitude of the $80-\mathrm{Hz}$ ASSR. At the $40-\mathrm{Hz}$ rate, however, there was a further amplitude decrease going from one-ear multiple to two-ear multiple stimuli. Importantly, these interactions did not differ between the normal-hearing and SNHL groups. Although supportive of the multiple-ASSR technique, there are likely situations where it is more efficient to use single stimuli. Future studies are required to assess these interactions in infants with varying degrees and configurations of hearing loss.
\end{abstract}

\section{Introduction}

Auditory steady-state responses (ASSRs) have received much recent attention by clinicians and researchers for reviews, see $[1,2]$. ASSRs to stimuli modulated in the $70-110 \mathrm{~Hz}$ range (the " $80-\mathrm{Hz}$ " ASSR) have their generators primarily in auditory brainstem structures; in the $35-45 \mathrm{~Hz}$ range (the "40-Hz" ASSR), ASSR sources are primarily cortical in origin with brainstem contributions [3].

Most recent attention has focussed on the $80-\mathrm{Hz}$ ASSR, which will likely be recommended for routine clinical assessment of auditory threshold in young infants in the near future, possibly in place of the tone-evoked auditory brainstem response (ABR), which is the current goldstandard technique [4-6]. However, lack of appropriate normative and clinical data in infants currently limits the clinical use of the 80-Hz ASSR [5, 7, 8]. Research in the 1980s indicated the $40-\mathrm{Hz}$ ASSR is difficult to record in infants $[9,10]$; however, many studies have indicated the $40-\mathrm{Hz}$
ASSR can provide accurate estimates of thresholds in adults, for example, [11].

Similar to tone-ABR recordings, the ASSR may be recorded to single-frequency stimuli presented to one ear at a time. This is the "single-stimulus" ASSR method, for which many clinical data have been published, for reviews, see $[5,12,13]$. Alternatively, it is also possible to record ASSRs to multiple stimuli (i.e., several frequencies) presented simultaneously to one or both ears. Although clinical data for this technique are more limited (for reviews, see $[5,12,13]$ ), this "multiple" ASSR technique has the potential to gather more information in a shorter amount of time, thus speeding up test time [14-16]. If there are no interactions between responses when multiple stimuli are presented (i.e., amplitudes are not smaller when stimuli are presented together), then the time to record responses is simply reduced by the number of stimuli presented simultaneously [17]. Even if some amplitude reductions ("interactions") exist, the presentation of multiple simultaneous stimuli may still be 
more efficient than the presentation of a single stimulus provided that the reduction in amplitude is less than $1 / \sqrt{K}$, where $K$ is the number of stimuli presented at the same time $[14,18]$. If ASSR amplitudes are smaller due to interactions, more sweeps must be averaged to reduce the EEG noise sufficiently to detect these smaller responses. Because EEG noise decreases predictably by the square root of the number of sweeps averaged, we can use this to determine whether the increased number of sweeps required to detect a smaller multiple-ASSR amplitude is offset by the gain in information [16]. With multiple stimuli, $K$ times the information (e.g., the number of frequencies) in a given sweep is obtained compared to the single-stimulus technique, thus provided we do not need to average more sweeps than a factor of $\sqrt{K}$, the multiple-ASSR will be more efficient. For example, if ASSR amplitudes decrease by $50 \%$ due to interactions when recording responses to eight simultaneous stimuli (e.g., four frequencies in each ear), the presentation of multiple stimuli is still more efficient provided the ASSR amplitude at the frequency of interest is greater than $35 \%(1 / \sqrt{K})$ of the single-stimulus ASSR amplitude [18].

In adults, the presentation of multiple stimuli simultaneously does not cause a decrease in $80-\mathrm{Hz}$ ASSR amplitude when stimuli are presented at $60 \mathrm{~dB}$ SPL or less in one or both ears, provided that the carrier frequencies are separated by at least an octave $[14,15,19]$. However, for $75-80 \mathrm{~dB}$ SPL stimuli, statistically significant interactions between responses to stimuli are seen such that amplitudes in the 4 -stimulus multiple condition (4 frequencies to one ear) decrease to $52-58 \%$ of their amplitude in the single-stimulus condition $[14,20,21]$, with no further decrease in the 8 -stimulus multiple condition (4 frequencies to each ear) [21]. Despite this, when compared to the single-stimulus technique, the $80 \mathrm{~Hz}$ multiple-ASSR technique remains more efficient, at least in adults $[14,21]$. In contrast to adults, in young infants, the presentation of $60 \mathrm{~dB}$ SPL multiple stimuli simultaneously results in a significant decrease in $80-\mathrm{Hz}$ ASSR amplitude. The reasons for this are uncertain; however, the decreases are not enough to make the multipleASSR technique less efficient, at least for normal infants [18].

As with the $80-\mathrm{Hz}$ ASSR, the $40-\mathrm{Hz}$ ASSR shows no interactions when multiple stimuli are presented at low (30 dBHL) intensities [22]. However, in contrast to the 80$\mathrm{Hz}$ ASSR, the $40-\mathrm{Hz}$ ASSR shows significant interactions when multiple stimuli are presented at $60 \mathrm{~dB}$ SPL, decreasing to approximately $60 \%$ of their amplitude in the singlestimulus condition $[14,19,22]$. In further contrast to the 80 $\mathrm{Hz}$ ASSR, the $40-\mathrm{Hz}$ ASSR decreases by an additional 33\% when multiple stimuli is presented to both ears dichotically $[14,22]$. For higher stimulus intensities (75-80 dB SPL), 40$\mathrm{Hz}$ ASSR shows interactions similar to those for the $80-\mathrm{Hz}$ ASSR except that only the $40 \mathrm{~Hz}$ shows an additional decrease with dichotic stimulation $[14,22]$. Taking into account these amplitude reductions, the use of multiple stimuli for the 40$\mathrm{Hz}$ ASSR may only be more efficient when testing low and moderate intensities (no. $60 \mathrm{~dB} \mathrm{SPL}$ ) and only for single-ear stimulation [22].

Many studies have shown reasonably accurate estimates of behavioural thresholds using the $80-$ and $40-\mathrm{Hz}$ ASSRs to multiplestimuli for reviews, see $[5,11,12,23]$, and multiplestimulus ASSR systems are currently being marketed to clinicians for threshold assessment. However, no study has assessed single- versus multiple-stimulus ASSR interactions in individuals with sensorineural hearing loss (SNHL); thus, it is not known if the multiple-stimulus ASSR technique is more efficient compared to the single-stimulus ASSR when hearing loss is present. It is reasonable to hypothesize that there would be greater interactions when cochlear hearing loss is present due to broader cochlear filters [24]. If the multiple-stimulus ASSR interactions are too large, it may be more efficient to present single rather than multiple stimuli when assessing individuals with SNHL. The present study assessed multiple-ASSR interactions by recording 80 - and $40-\mathrm{Hz}$ ASSRs to single versus multiple stimuli in adult groups with either normal hearing or SNHL.

\section{Methods}

2.1. Participants and Procedure. A total of 24 adults participated in this study: 12 with normal hearing aged between 23 and 63 years (mean age $38.2 \pm 13.0$ yrs; 6 female) and 12 with SNHL aged between 22 and 69 years (mean age $52.3 \pm 15.9 \mathrm{yrs} ; 7$ female). The normal-hearing group had pure-tone behavioural thresholds of $20 \mathrm{~dB}$ HL (ANSI, S3.6-1996) or better in both ears for 500, 1000, 2000, and $4000 \mathrm{~Hz}$. Their average (and standard deviation, SD) hearing thresholds were 8 (6.2), 5 (6.6), 5 (6.6), and 7 (8.1) dB HL, respectively for $500,1000,2000$, and $4000 \mathrm{~Hz}$ in the test ear. The participants with SNHL had pure-tone behavioural thresholds for their test ear greater than $20 \mathrm{~dB}$ HL for at least one of $500,1000,2000$, and $4000 \mathrm{~Hz}$, with boneconduction thresholds within $5 \mathrm{~dB}$ of thresholds for airconduction stimuli (i.e., no conductive or mixed hearing loss). However, because ASSR stimuli were presented at $80 \mathrm{~dB}$ HL, participants with SNHL had to have a threshold of $65 \mathrm{~dB}$ HL or better for at least one of 1000 or $2000 \mathrm{~Hz}$ in the test ear. Mean (SD) test-ear thresholds for the SNHL group were: 30 (15), 36 (16), 47 (12), and 53 (16) dB HL for 500, 1000, 2000, and $4000 \mathrm{~Hz}$ respectively. Mean (SD) thresholds for their nontest ear were 33 (19), 44 (25), 50 (22), and 59 (23) dB HL. Table 1 shows the test-ear hearing thresholds for individual SNHL participants.

Subject eligibility criteria excluded participants with any of the following: (i) age greater than 70 years; (ii) external/ middle ear involvement (e.g., ear infection) in either ear; (iii) neurological involvement (e.g., cerebral palsy, multiple sclerosis); (iii) 80 - or $40-\mathrm{Hz}$ ASSR absent in monotic single conditions for either 1000 or $2000 \mathrm{~Hz}$. (As responses in the MS condition typically show the largest amplitudes, this criterion ensured (i) the ability to assess the effects of going from single to multiple stimuli and (ii) results for all subjects were available for all conditions. Two additional subjects were excluded due to this criterion.) Finally, (iv) subjects were rejected (i.e., considered "noisy") if two ASSR recordings out of three reached the limit of 60 sweeps before reaching noise criteria (see below for description of the noise criteria). There were three additional subjects rejected for 
TABle 1: Pure-tone behavioural hearing thresholds (test ear, in $\mathrm{dBHL}$ ) of the SNHL group.

\begin{tabular}{lcccc}
\hline Subj. no. & $500 \mathrm{~Hz}$ & $1000 \mathrm{~Hz}$ & $2000 \mathrm{~Hz}$ & $4000 \mathrm{~Hz}$ \\
\hline SNHL1 & 30 & 35 & 45 & 35 \\
SNHL2 & 10 & 20 & 25 & 30 \\
SNHL3 & 25 & 40 & 40 & 40 \\
SNHL4 & 40 & 55 & 60 & 45 \\
SNHL5 & 35 & 30 & 35 & 60 \\
SNHL6 & 65 & 65 & 65 & 65 \\
SNHL7 & 25 & 45 & 45 & 60 \\
SNHL8 & 35 & 50 & 60 & 90 \\
SNHL9 & 20 & 35 & 50 & 50 \\
SNHL10 & 20 & 20 & 35 & 40 \\
SNHL11 & 15 & 10 & 50 & 60 \\
SNHL12 & 40 & 30 & 50 & 60 \\
\hline Mean & 30 & 36 & 47 & 53 \\
SD & 15 & 16 & 12 & 16 \\
\hline
\end{tabular}

SD: standard deviation.

"noisy" results and therefore not included in the data for calculation of final results.

Informed written consent, approved by The University of British Columbia Behavioural Research Ethics Board, was obtained before commencing the study and the participants were paid an honorarium at the end of the session. Testing was performed in a double-walled sound-attenuating booth. The session began with pure-tone behavioural audiometry for air- and bone-conduction stimuli. 40-Hz ASSR results were then obtained, with the subjects typically watching a muted DVD movie with subtitles, followed by the recording of the $80-\mathrm{Hz}$ ASSR with the subject reclined in a comfortable chair and instructed to relax or sleep. Four conditions were recorded: (i) dichotic multiple (DM): both ears simultaneously, all four frequencies $(500,1000,2000$, and $4000 \mathrm{~Hz}$ ); (ii) monotic multiple: test ear only, all four frequencies simultaneously; (iii) monotic single for $1000 \mathrm{~Hz}$ : test ear only; (iv) Monotic Single for $2000 \mathrm{~Hz}$ : test ear only. The recording order of the conditions was randomized. The test ear was chosen according to the following criteria: (i) randomized, if symmetrical hearing loss or (ii) for asymmetrical hearing loss, the ear with smaller difference between 1000 and $2000 \mathrm{~Hz}$ thresholds.

2.2. ASSR Stimulus Parameters. The stimuli for the 80 - and $40-\mathrm{Hz}$ ASSR were generated by the Rotman MultiMASTER research system [25] with carrier frequencies of 500, 1000, 2000 , and $4000 \mathrm{~Hz}$. Using a digital-to-analog conversion rate of $31,250 \mathrm{~Hz}$ [26], stimuli were sinusoidally amplitude (100\%) and frequency (25\%) modulated (i.e., AM/FM stimuli), for the $80-\mathrm{Hz}$ ASSR at 77.15, 84.96, 92.77, and $100.59 \mathrm{~Hz}$ (left ear) and 81.06, 88.87, 96.68, and 105.47 (right ear), for 500, 1000, 2000, and 4000, respectively. Modulation rates for the 40-Hz ASSR were 35.94, 39.06, 42.19, and $45.31 \mathrm{~Hz}$ (left ear) and 37.5, 40.62, 43.75, and $46.87 \mathrm{~Hz}$ (right ear). The modulation rates were chosen to ensure each EEG recording contained an integer number of modulation cycles and each recording sweep contained an integral number of carrier frequencies [25].

The stimuli were calibrated using a Larson Davis model 824 sound-level meter (using "peak SPL" minus $3 \mathrm{~dB}$ to determine peak-to-peak equivalent SPL). The four AM/FM tones were each calibrated separately in $\mathrm{dB}$ HL and then combined. The intensity of the individual stimuli was kept constant at $80 \mathrm{~dB}$ HL for all conditions tested. The stimuli were presented via air conduction through ER-3A insert earphones in both ears. Both earphones were kept on throughout the recording session.

2.3. ASSR Recording Parameters. ASSRs were recorded using the Rotman MultiMASTER research system. Three goldplated electrodes were used to record the electrophysiologic responses at (i) $\mathrm{Fz}$ (noninverting), (ii) midline at the nape (inverting), and (iii) left mastoid (ground). All interelectrode impedances were kept below $3 \mathrm{kOhms}$ at $10 \mathrm{~Hz}$. The EEG was amplified 80,000 times and filtered using a bandpass of 30 to $250 \mathrm{~Hz}(12 \mathrm{~dB} /$ octave $)$ for the 80 $\mathrm{Hz}$ ASSR and a bandpass of 5 to $100 \mathrm{~Hz}$ for the $40-\mathrm{Hz}$ ASSR [11]. A $1250 \mathrm{~Hz}$ analog-to-digital conversion rate was used [27]. Each EEG recording sweep lasted 13.107 seconds and was comprised of 16 epochs of 1024 data points each (0.8192 seconds per epoch). Artifact rejection was set to eliminate epochs of electrophysiological activity with amplitudes exceeding $\pm 60 \mu \mathrm{V}$ for the 80 - $\mathrm{Hz}$ ASSR and $\pm 80 \mu \mathrm{V}$ for the $40-\mathrm{Hz}$ ASSR.

ASSRs were averaged in the time domain and then analyzed online into the frequency domain using a fast Fourier transform (FFT). In order to decrease the effect of EEG noise, weighted averaging (80-Hz ASSR: 70-110; 40-Hz ASSR: $30-50 \mathrm{~Hz}$ ) was used, for further details, see [28]. The $F$-ratio was calculated by MultiMASTER and estimated the probability that the amplitude of the ASSR at the modulation frequency for each carrier frequency was significantly different from the amplitude of the background noise in adjacent frequencies within \pm 60 bins of the modulation frequency ("mean noise") [25]. Recordings were automatically stopped if significant responses were not reached within 60 sweeps. Recordings always continued for a minimum of 12 sweeps and continued until the noise criteria were met for all carrier frequencies (test ear). If the mean noise after a maximum of 60 sweeps did not drop below the noise criteria of $16.5 \mathrm{nV}$ for the $80-\mathrm{Hz}$ ASSR [21] and $33 \mathrm{nV}$ for the $40-\mathrm{Hz}$ ASSR $[11,13,20,22]$, the condition was repeated (see above for subject eligibility criteria). Although not a stopping criterion, response significance $(P<.05)$ was also noted.

2.4. Statistical Analyses. In addition to descriptive statistics (mean, SD, etc.), amplitudes were analyzed, separately for the 80 - and 40-Hz ASSRs, using a mixed-model analysis of variance (ANOVA) ( 1 between-subjects factor ( 2 groups), 2 within-subjects factors ( 3 conditions $\times 2$ stimulus frequencies)). To compare results between $80-$ and $40-\mathrm{Hz}$ ASSRs, amplitudes were normalized to a percentage of the amplitude in the MS condition and assessed using a mixed-model analysis of variance (ANOVA) (1 between-subjects factor 

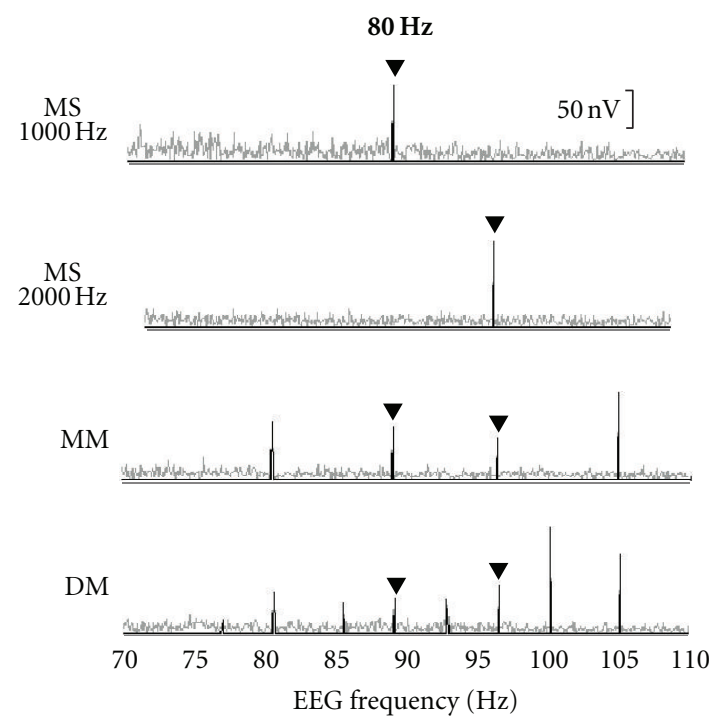
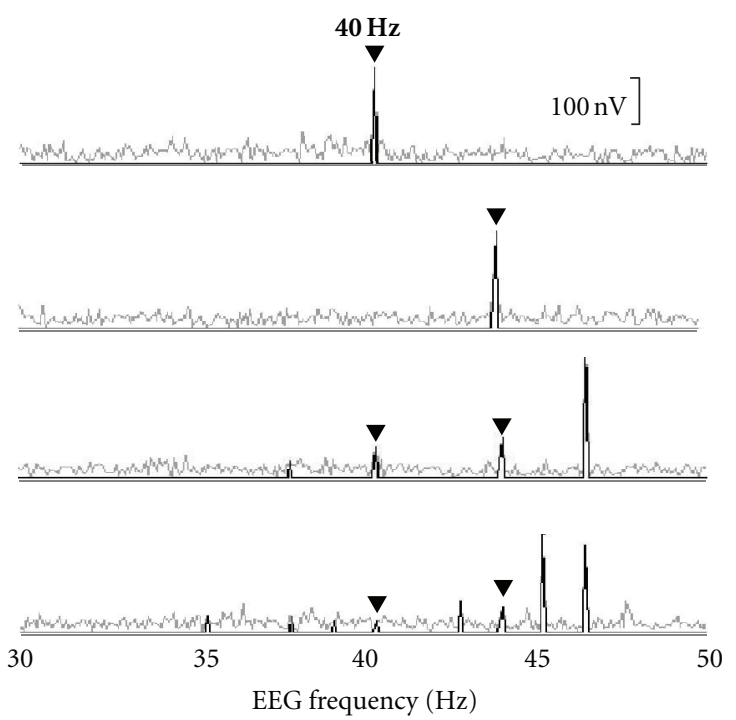

FIGURE 1: Representative normal-hearing subject's ASSR amplitude spectra in the monotic single (MS), monotic multiple (MM), and dichotic multiple (DM) stimulus conditions. Filled triangles indicate response to 1000 and $2000 \mathrm{~Hz}$ stimuli.

(2 groups), 3 within-subjects factors ( 2 rates, 2 conditions, 2 stimulus frequencies)).

Differences in the above mixed-model ANOVAs were considered significant if $P<.05$. Newman-Keuls post hoc comparisons were performed for significant $(P<.05)$ main effects and interactions.

Percent-amplitude results allowed us to assess the efficiency of the single versus multiple conditions. Even if significant decreases in ASSR amplitudes with presentation of multiple stimuli are seen, this does not necessarily mean the multiple-stimulus condition is less efficient than the single-stimulus condition. Relative efficiency is a measure that considers the increase in information relative to the decrease in amplitude when going from single- to multiplestimulus conditions. Provided the reduction in amplitude is less than $1 / \sqrt{K}$, where $K$ is the number of stimuli presented simultaneously, the multiple stimulus condition remains more efficient $[14,18,22]$. (For a full description of relative efficiency, see Hatton and Stapells [18].) Thus, the 4-stimulus MM condition is more efficient than the MS condition provided its amplitude is more than $50 \%$ of the MS condition's amplitude. Similarly, the 8-stimulus DM condition is more efficient if its amplitude is more than $35 \%$ of that of the MS condition. Relative efficiency (RE) was calculated using the following formula: $\mathrm{RE}=(\mathrm{AMPm} / \mathrm{AMPs}) * \sqrt{K}$, where RE is the relative efficiency; AMPmis the individual subject's amplitude for specific multiple condition; AMPs is the individual subject's amplitude for same frequency in the MS condition; $K$ is the number of simultaneous stimuli.

Multiple-stimulus conditions with RE values $>1$ are more efficient than the MS condition; those with $\mathrm{RE}$ values $<1$ are less efficient. In the present study, dependent-sample $t$ tests were carried out to determine whether RE results in a multiple-stimulus condition were significantly different from the corresponding MS condition, where RE always equals "1". After Bonferroni correction for multiple $t$-tests, results were considered significant if $P<.00625$ (alpha level of .05 divided by 8 tests $=.00625$ ).

\section{Results}

Typical results for a normal-hearing subject and a subject with SNHL are shown in Figures 1 and 2, respectively. Across all conditions, $40-\mathrm{Hz}$ ASSR amplitudes are much larger than $80 \mathrm{~Hz}$ amplitudes for both subjects. The effects of single versus multiple stimuli, however, differ between the two rates. For both subjects, going from single (MS) to multiple (MM and DM) stimuli results in a decrease in $80-\mathrm{Hz}$ ASSR amplitude. However, going from multiple one ear (MM) to multiple two ears (DM) does not further reduce the amplitude of the $80-\mathrm{Hz}$ ASSR. In contrast, at the $40 \mathrm{~Hz}$ rate, in addition to a large amplitude decrease going from single (MS) to multiple one ear (MM), going to one-ear multiple to two-ear multiple (DM) results in a further decrease in amplitude. It is this latter decrease that is particularly different between 80 - and $40-\mathrm{Hz}$ ASSRs.

3.1. 80-Hz ASSR Amplitudes. Figure 3 shows the mean ASSR amplitudes for the three conditions (MS, MM, DM), for both groups (normal hearing and the SNHL), for both frequencies $(1000$ and $2000 \mathrm{~Hz})$, and for both rates $(80$ and $40 \mathrm{~Hz})$.

As can be seen in the left panels of Figure 3, there were no differences in the $80 \mathrm{~Hz}$ response amplitudes for the two groups, with a mixed-model ANOVA revealing no significant main effects or interactions involving "group" (group: $P=.651$; condition $\times$ group: $P=.757$; frequency $\times$ group: $P=.072$; condition $\times$ frequency $\times$ group: $P=$ $.404)$. The frequency $\times$ group trend $(P=.072)$ was due to the larger amplitude (albeit not significant) for $1000 \mathrm{~Hz}$ $(97.5 \mathrm{nV})$ compared to $2000 \mathrm{~Hz}(79.6 \mathrm{nV})$ in the SNHL group; this difference was not seen in the normal-hearing group (1000 Hz: $82.6 \mathrm{nV}$; $2000 \mathrm{~Hz}$ : $81.9 \mathrm{nV}$ ). 


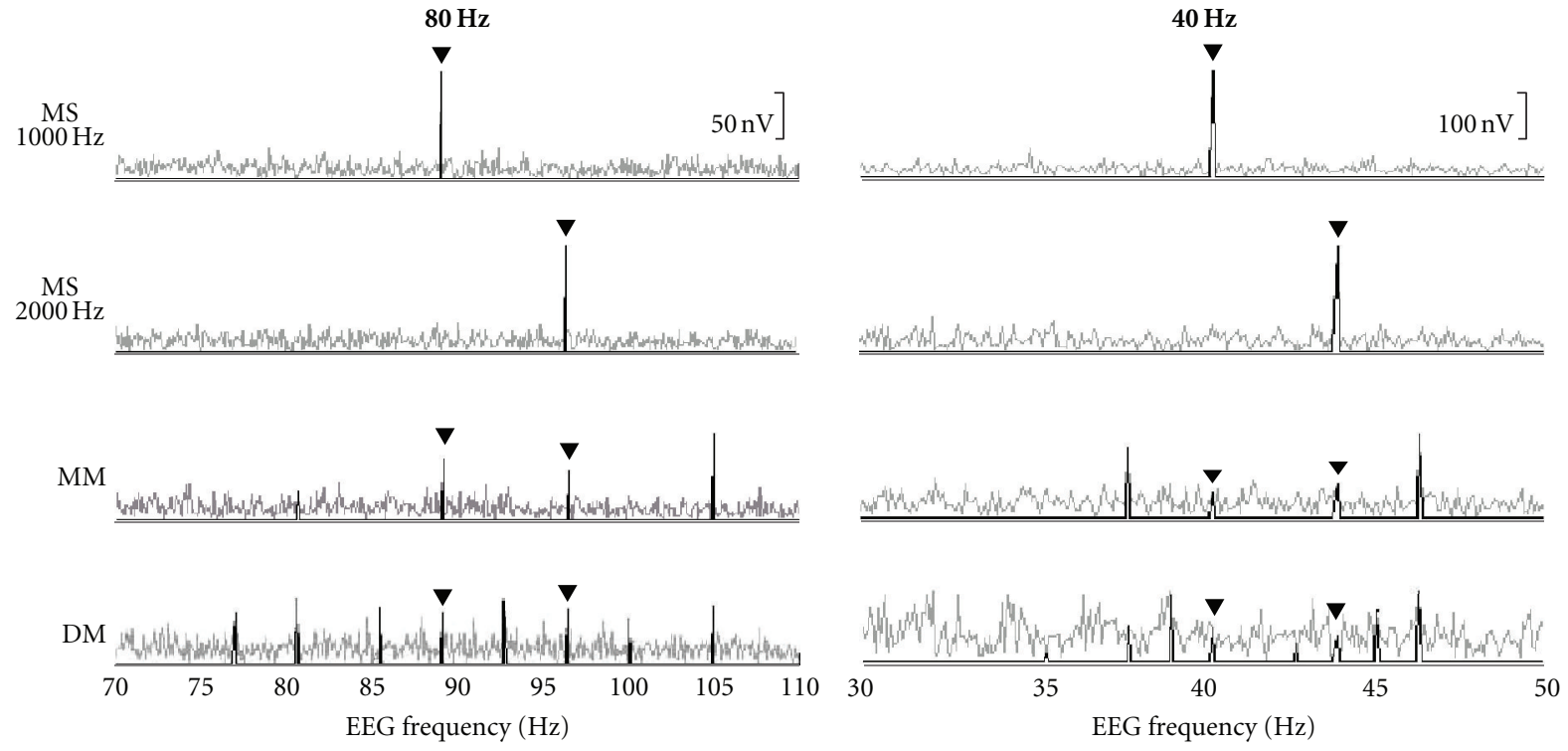

Figure 2: Representative SNHL subject's ASSR amplitude spectra in the MS, MM, and DM stimulus conditions. Filled triangles indicate response to $1000-$ and $2000-\mathrm{Hz}$ stimuli.

As would be expected from previous studies of the effects of single versus multiple stimuli presented at higher intensities $[14,19,20,29]$, Figure 3 shows that multiple stimuli resulted in significant reduction in the amplitudes of the $80-\mathrm{Hz}$ ASSR. The above ANOVA showed a significant main effect of condition $(P<.001)$. Post hoc analysis revealed that the amplitudes in the MM and DM conditions were significantly smaller $(P<.001)$ than those of the MS condition, with no difference between the MM and the DM conditions $(P=.501)$. Across all conditions, there was no significant main effect of frequency $(P=.054)$. However, as indicated by a significant condition $\times$ frequency interaction $(P=.015)$, the $80-\mathrm{Hz}$ ASSR amplitude for $1000 \mathrm{~Hz}$ was significantly larger than the $2000 \mathrm{~Hz}$ amplitude in the MS condition $(146.8$ versus $122.9 \mathrm{nV} ; P<.001)$; this difference disappeared in the multiple conditions $(P=.202-$ .306).

3.2. 40-Hz ASSR Amplitudes. As can be seen in the right panels of Figure 3, there were no differences in the $40-\mathrm{Hz}$ ASSR amplitudes between the two groups, with a mixedmodel ANOVA revealing no significant main effect of group $(P=.880)$ and no significant interactions involving group $(P=.312-.641)$.

Similar to previous studies on the $40-\mathrm{Hz}$ ASSR [14, 20, $22,30]$ of the effects of single versus multiple stimuli, the right panel of Figure 3 shows that multiple simultaneous stimuli resulted in significant reductions in the amplitudes of the 40-Hz ASSR. In a mixed-model ANOVA with the same design as that for the $80-\mathrm{Hz}$ ASSR results, a significant main effect for condition $(P<.001)$ was found, such that 40 $\mathrm{Hz}$ ASSR amplitude in the MS condition $(416.0 \mathrm{nV}$, pooled across groups and frequencies) was significantly $(P<.001)$ reduced to $153.5 \mathrm{nV}$ in the MM condition, with a further significant $(P=.048)$ amplitude reduction in the DM condition $(101.5 \mathrm{nV})$. There were no significant interactions involving condition $(P=.294-.381)$.

As would be expected from the literature for review, see [2], response amplitudes for the $40-\mathrm{Hz}$ ASSR were significantly larger for $1000 \mathrm{~Hz}(237.7 \mathrm{nV})$ compared to $2000 \mathrm{~Hz}(209.6 \mathrm{nV}$; main effect of frequency: $P=.042)$. There were no significant interactions involving frequency $(P=.340-.641)$.

3.3. Comparison of the 80 and $40 \mathrm{~Hz}$ Results Change in Amplitude from $M S$ to the $M M$ and DM Conditions. As previous studies have shown for review, see [2] and also obvious in Figure 3, the $40-\mathrm{Hz}$ ASSR amplitudes are 2 to 3 times larger than those of the $80-\mathrm{Hz}$ ASSR. To compare results between both rates, amplitudes were normalized to a percentage of the amplitude in the MS condition. Table 2 shows the percent amplitude results, as well as the relative efficiency results, for the MM and DM conditions.

Overall, a mixed-model ANOVA showed a significant rate main effect $(P=.003)$, with $40-\mathrm{Hz}$ ASSR percent amplitude being smaller (80 Hz: 49.3\%; $40 \mathrm{~Hz}: 36.4 \%$ ), indicating a greater impact of multiple simultaneous stimuli on the $40-\mathrm{Hz}$ ASSR. The exception to this was for the SNHL group at $1000 \mathrm{~Hz}$, where the $40-\mathrm{Hz}$ ASSR percent amplitude was the same as the $80 \mathrm{~Hz}$ (rate $\times$ frequency $\times$ group interaction: $P=.032$ ). Across both groups, rates, and frequencies, the DM condition shows significantly smaller percent amplitudes than the MM (MM: 45.3\%, DM: 40.4\%; condition main effect: $P=.049)$. However, the smaller percentage for DM was due to a significant decrease in the $40-\mathrm{Hz}$ ASSR percent amplitude; no change occurred in the $80-\mathrm{Hz}$ ASSR percent amplitude (rate $\times$ condition interaction: $P<.001$ ). Thus, $40-\mathrm{Hz}$ ASSR amplitude decreases further with 2-ear multiple stimuli whereas 80 $\mathrm{Hz}$ ASSR shows no additional decrease; both rates showed 

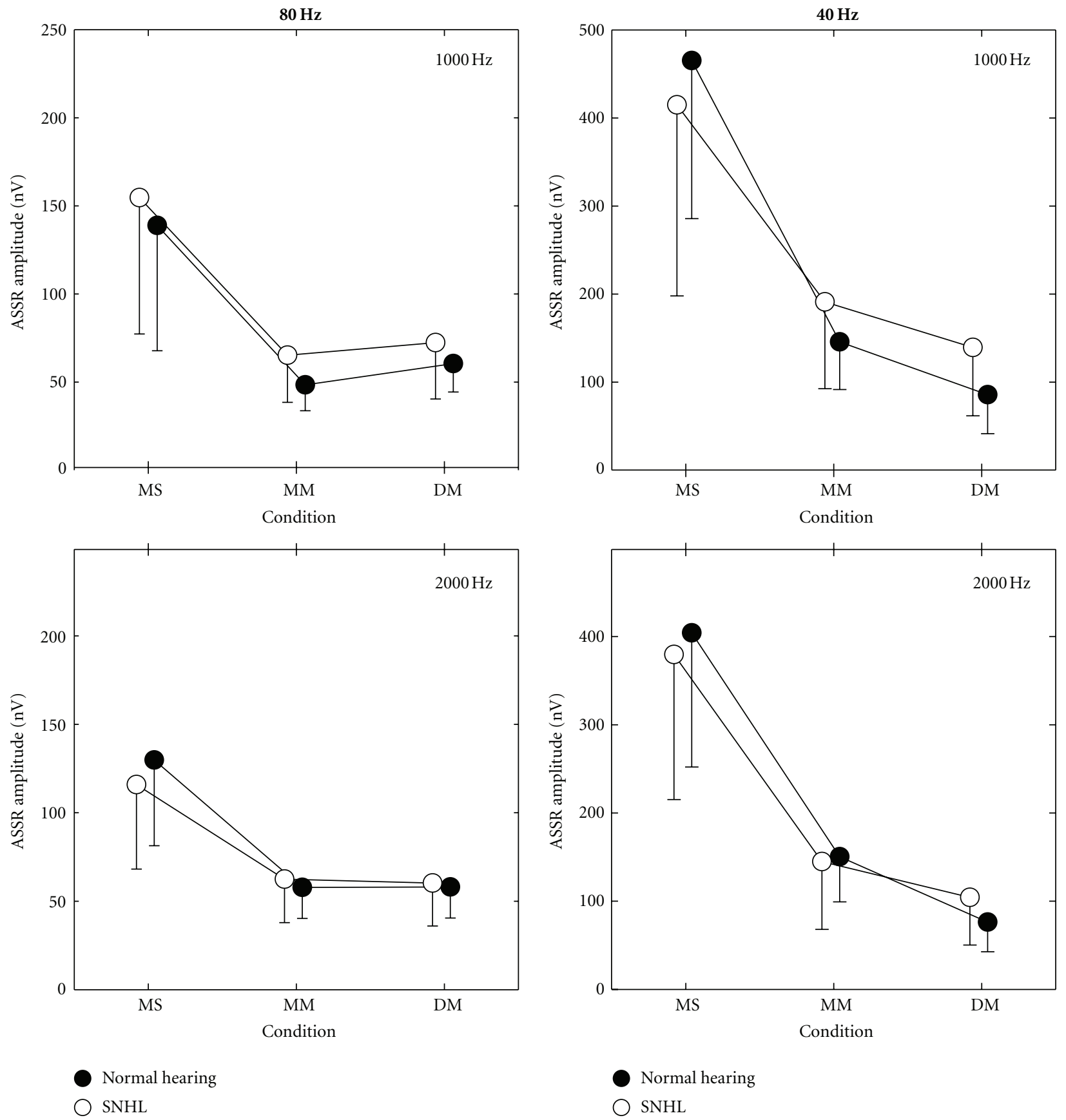

FIgURE 3: Mean (and SD) ASSR response amplitudes for both groups for the MS, MM, and DM conditions at the 40 and $80 \mathrm{~Hz}$ rates.

similar percent decreases in the MM condition relative to the MS condition (rate $\times$ condition interaction: $P<.001$ ). With the exception noted above (SNHL 40-Hz ASSR percent amplitude at $1000 \mathrm{~Hz}$ ), there were no significant main effects or interactions involving groups or stimulus frequency.

As the results in Table 2 indicate, there are many amplitude means (in \% of MS amplitude) that are smaller than $50 \%$ in the 4 -stimulus MM condition. To be more efficient, however, amplitude should not decrease more than $50 \%[1 / \sqrt{4}=0.5]$. The "efficiency" of a test is quantified by the calculation of "relative efficiency (RE)," the mean values of which are also shown in Table 2. Across both groups, the MM condition shows RE values less than or close to one (0.84-1.03) for the $80-\mathrm{Hz}$ ASSR, suggesting single stimuli would be more (or at least, equally) efficient than the MM condition. However, $80-\mathrm{Hz}$ ASSR results for the 8-stimulus DM condition show RE values well above one ( 1.5) indicating DM is more efficient than both onestimulus (MS) or four-stimulus (MM) conditions. For the $40-\mathrm{Hz}$ ASSR, similar RE results were seen for the MM condition; however, the large decrease in amplitude for 8stimulus 2-ear DM condition results in lower RE values (0.71-0.92). One oddity, however, were the relatively high $40-\mathrm{Hz}$ ASSR percent amplitudes and, thus, high RE values 
TABLE 2: Percent amplitude and relative efficiency.

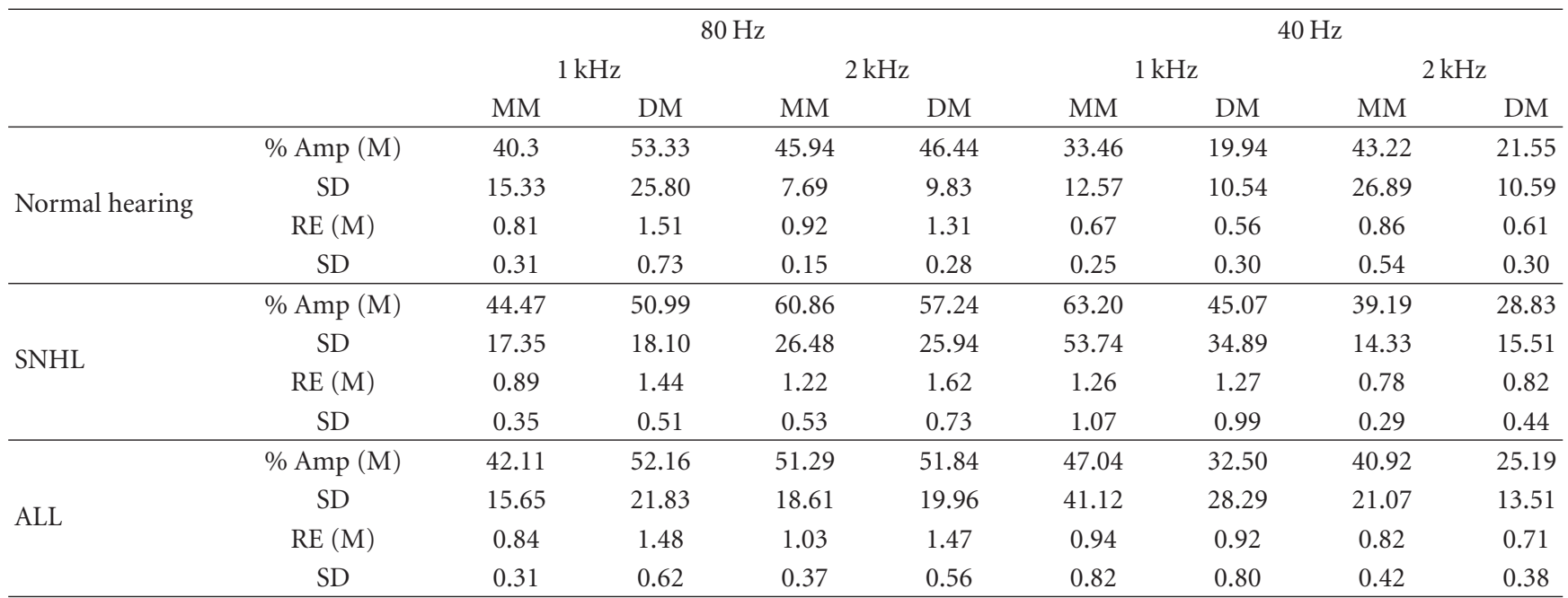

Percent amplitude and RE calculated relative to each subject's results in the MS condition.

M: mean; SD: standard deviation; RE: relative efficiency.

seen in MM (1.26) and DM (1.27) conditions for the SNHL group's $1000-\mathrm{Hz}$ results.

Dependent sample $t$-tests were carried out to determine whether RE results in a multiple condition (MM or DM) were significantly different from the single-stimulus condition (MS). The $80-\mathrm{Hz}$ ASSR DM condition was significantly more efficient at both $1000(P<.001)$ and $2000 \mathrm{~Hz}(P<.001)$ than the corresponding MS condition. In contrast, the $40-\mathrm{Hz}$ ASSR DM condition at $2000 \mathrm{~Hz}$ was significantly less efficient $(P=.001)$ than the corresponding MS condition. The five other comparisons indicated RE values for $\mathrm{MM}$ or $\mathrm{DM}$ conditions were not significantly different $(P=.021-728)$ from the MS single condition.

\section{Discussion}

The current study investigated the effect of single versus multiple simultaneous stimuli on the $80-$ and $40-\mathrm{Hz}$ ASSRs in individuals with normal hearing or SNHL. Results show significant interactions (i.e., decreased amplitude) for both $80-$ and $40-\mathrm{Hz}$ ASSRs going from single (MS) to multiple (MM) stimuli in one ear. Going from multiple one ear (MM) to multiple two ears (DM) does not further reduce the amplitude of the $80-\mathrm{Hz}$ ASSR, whereas at the $40 \mathrm{~Hz}$ rate, there is a further amplitude decrease going from oneear multiple (MM) to two-ear multiple (DM) stimuli. These interactions were the same in both normal-hearing and SNHL groups.

The finding of similar interactions between the normalhearing and SNHL groups was not expected. Because individuals with SNHL have broader cochlear filters, we hypothesized the SNHL group would show larger amplitude decreases with multiple stimuli (i.e., more interactions). This result could be due to the use of the $80 \mathrm{~dB} H \mathrm{HL}$ stimulus intensity, which would result in relatively broad cochlear activation for both groups, and thus, interaction would be similar. It is not possible to test lower intensities (where interactions would be smaller) in individuals with SNHL as such levels would be subthreshold. (Although the SNHL group was, on average, 14 years older than the normal-hearing group, this cannot explain the lack of difference between groups. First, the age difference is not large enough to affect the ASSR, and second, if anything, one would expect smaller $80-\mathrm{Hz}$ ASSR amplitudes in the older subjects [31], which, although not significant, was the opposite to the present study's results.) Although the current study's results may suggest that interactions are not a greater concern in individuals with SNHL, further research is needed to investigate the interactions at other frequencies and intensities.

The finding of significant interactions for the $80-\mathrm{Hz}$ ASSR to multiple stimuli (MM and DM) for higher intensity stimuli $(75-80 \mathrm{dBHL})$ is consistent with previous studies $[14,20,21,29]$. The source of the interactions for the 80$\mathrm{Hz}$ ASSRs is not yet known but reflects cochlear and/or brainstem mechanisms $[3,17]$. Clinically, these interactions increase the number of averages required to reach given signal-to-noise ratio. This increased time may be offset by obtaining responses to multiple stimuli simultaneously. The relative efficiency measure provides a quantification of this trade off. In the current study, the relative efficiency of the 80-Hz ASSR for the one-ear, four-stimuli, multiple-stimulus condition (MM) was not significantly different from the single-stimulus (MS) condition. In contrast, testing both ears simultaneously with four stimuli (DM) was significantly more efficient than MS. From this, one might conclude that the multiple-stimulus $80-\mathrm{Hz}$ ASSR is more efficient than the MS condition providing two ears are tested simultaneously. However, the multiple-ASSR may not be more efficient when significant differences between ears and/or frequencies exist, in which case perhaps it might be best to do single stimuli especially if only one ear is being tested at a time.

The interaction for the $40-\mathrm{Hz}$ ASSR for single versus multiple stimuli in one ear is such that relative efficiency 
values for the MM condition are not significantly better than the MS condition, similar to the $80-\mathrm{Hz}$ ASSR. In contrast to the $80-\mathrm{Hz}$ ASSR, however, relative efficiency values for the multiple two-ear (DM) condition are significantly lower than the MS condition for the $40-\mathrm{Hz}$ ASSR, at least for high intensities; therefore, it does not appear that multiple simultaneous stimuli are more efficient for the $40-\mathrm{Hz}$ ASSR. At lower intensities (30 and $55 \mathrm{dBHL}$ ), however, previous research showed that both the MM and DM conditions are more efficient than the MS condition for the 40-Hz ASSR, at least for normal-hearing subjects [22]. Similar to the $80-\mathrm{Hz}$ ASSR, however, the $40-\mathrm{Hz}$ ASSR to multiple stimuli may not be more efficient when significant differences between ears and/or frequencies exist.

The finding of smaller $40-\mathrm{Hz}$ ASSR interactions for the SNHL group at $1000 \mathrm{~Hz}$ may reflect either (i) a cochlear phenomenon, whereby there is a decreased influence of $2000 \mathrm{~Hz}$ on the $1000-\mathrm{Hz}$ responses due to greater hearing loss at $2000 \mathrm{~Hz}$, and/or (ii) a cortical phenomenon, with plasticity-related enhancement of function in the cortical areas representing $1000 \mathrm{~Hz}$ due to the hearing loss present at $2000 \mathrm{~Hz}$. There are many reports in the literature indicating cortical plasticity enhancing responses to frequencies at the edge of sloping hearing loss for review, see [32]. The fact that the $80-\mathrm{Hz}$ ASSR, which reflects cochlear and brainstem processes, did not show this pattern suggests the relatively smaller interactions for the $40-\mathrm{Hz}$ ASSR at $1000 \mathrm{~Hz}$ for the SNHL group do not originate from cochlear processes.

Audiological use of the $40-\mathrm{Hz}$ ASSR is of particular interest for threshold estimation in adults undergoing assessment for medicolegal and/or compensation purposes. The 80$\mathrm{Hz}$ ASSR is smaller in amplitude, for review, see [2], and requires significantly longer test times [11]. Although most studies have focused on the $40-\mathrm{Hz}$ ASSR to single stimuli, the multiple-stimulus technique has also shown excellent threshold estimation using the $40-\mathrm{Hz}$ ASSR in adults with hearing loss [11]. Results of the present study suggest that when using multiple stimuli for the $40-\mathrm{Hz}$ ASSR, each ear should be tested separately (i.e., MM). Because the $40-\mathrm{Hz}$ ASSR is difficult to record in sleeping infants [9, 10, 33], clinical applications of the ASSR in infants have focused on the $80-\mathrm{Hz}$ ASSR. The results of the present study suggest twoear testing, that is, (DM) would be more efficient for $80-\mathrm{Hz}$ ASSR.

There are several limitations to the present study. First, the stimulus intensity was limited to $80 \mathrm{dBHL}$. Lower intensities (e.g., $60 \mathrm{dBHL}$ ) would have made it difficult to test individuals with hearing loss. Testing at higher intensities (e.g., 90-100 dBHL) would allow a greater range of hearing losses, but also entail issues concerning loudness discomfort and possibly risk of additional hearing loss due to overstimulation. A second limitation is the relatively narrow range of degrees and configurations of hearing loss in the SNHL group, with most subjects having mild-to-moderate slightly sloping hearing loss. In order to have tested more significant hearing loss, we would have had to use a stimulus intensity higher than $80 \mathrm{dBHL}$ to ensure we get a response in at least the MS condition. A third limitation is we only looked at interactions for 1000 and $2000 \mathrm{~Hz}$ stimuli. A primary reason why we restricted the study to these frequencies was that the additional test frequencies (e.g., 500 and/or $4000 \mathrm{~Hz}$ ) would significantly increase the test time for each subject. Further, testing at $4000 \mathrm{~Hz}$ would have required a higher intensity due to the subjects' sloping hearing loss. A fourth limitation is that this study assessed interactions in adults with hearing loss; however, the group of primary interest for clinical testing is infants with hearing loss.

\section{Conclusions}

The current study shows that ASSR interactions with $80 \mathrm{dBHL}$ stimuli, which result in smaller amplitudes when going from single to multiple stimuli, do not differ between adults with normal hearing and SNHL. This suggests that the multiple-ASSR technique is not less efficient in subjects with SNHL compared to those with normal hearing. Previous research has suggested the multiple-stimulus $80-\mathrm{Hz}$ ASSR technique is more efficient than the single-stimulus ASSR in normal infants [18]. Although supportive of the multipleASSR technique, there are likely situations where it is more efficient to use single stimuli. Future studies are required to assess these interactions in infants with varying degrees and configurations of hearing loss.

\section{Acknowledgments}

The authors thank the audiologists, staff, and clients from WorkSafeBC and the Western Institute for the Deaf and Hard of Hearing for their participation and assistance with this research. This research was supported by grants to the second author from the Natural Sciences and Engineering Research Council (NSERC) of Canada and the Canadian Institutes of Health Research (CIHR).

\section{References}

[1] G. Rance, The Auditory Steady State Reponse: Generation, Recording, and Clinical Application, Plural Publishing, San Diego, Calif, USA, 2008, edited by G. Rance.

[2] T. W. Picton, Human Auditory Evoked Potentials, Plural Publishing, San Diego, Calif, USA, 2011.

[3] A. T. Herdman, T. W. Picton, and D. R. Stapells, "Place specificity of multiple auditory steady-state responses," Journal of the Acoustical Society of America, vol. 112, no. 4, pp. 15691582, 2002.

[4] American Speech-Language-Hearing Association, "Guidelines for the audiologic assessment of children from birth to 5 years of age," American Speech-Language-Hearing Association, vol. 2012, 2004.

[5] D. R. Stapells, "Frequency-specific threshold assessment in young infants using the transient ABRand the brainstem ASSR," in Comprehensive Handbook of Pediatric Audiology, R. C. Seewald and A. M. Tharpe, Eds., pp. 409-448, Plural Publishing, San Diego, Calif, USA, 2011.

[6] British Columbia Early Hearing Program (BCEHP), "BCEHP audiology assessment protocol,” 2012, http://www.phsa.ca/ AgenciesAndServices/Services/BCEarlyHearing/ForProfessionals/Resources/Protocols-Standards.htm. 
[7] J. Busa, J. Harrison, J. Chappell et al., "Year 2007 position statement: principles and guidelines for early hearing detection and intervention programs," Pediatrics, vol. 120, no. 4, pp. 898 921, 2007.

[8] S. A. Small and A. Dimitrijevic, "Physiological mechanisms underlying auditory steady-state responses (ASSRs)," in Translational Perspectives in Auditory Neuroscience: Hearing across the Lifespan-Assessment and Disorders, K. L. Tremblay and R. Burkard, Eds., Plural Publishing, San Diego, Calif, USA, 2012.

[9] T. Suzuki and K. Kobayashi, "An evaluation of 40-Hz eventrelated potentials in young children," Audiology, vol. 23, no. 6, pp. 599-604, 1984.

[10] D. R. Stapells, R. Galambos, J. A. Costello, and S. Makeig, "Inconsistency of auditory middle latency and steady-state responses in infants," Electroencephalography and Clinical Neurophysiology, vol. 71, no. 4, pp. 289-295, 1988.

[11] A. van Maanen and D. R. Stapells, "Comparison of multiple auditory steady-state responses (80 versus $40 \mathrm{~Hz}$ ) and slow cortical potentials for threshold estimation in hearingimpaired adults," International Journal of Audiology, vol. 44, no. 11, pp. 613-624, 2005.

[12] A. I. Tlumak, E. Rubinstein, and J. D. Durrant, "Meta-analysis of variables that affect accuracy of threshold estimation via measurement of the auditory steady-state response (ASSR)," International Journal of Audiology, vol. 46, no. 11, pp. 692-710, 2007.

[13] B. Cone and A. Dimitrijevic, "The auditory steady-state response," in Handbook of Clinical Audiology, J. Katz, L. Medwetsky, R. Burkard, and L. Hood, Eds., pp. 322-350, Lippingcott, Williams \& Wilkins, Baltimore, Md, USA, 2009.

[14] M. S. John, O. G. Lins, B. L. Boucher, and T. W. Picton, "Multiple auditory steady-state responses (MASTER): stimulus and recording parameters," Audiology, vol. 37, no. 2, pp. 59-82, 1998.

[15] A. T. Herdman and D. R. Stapells, "Thresholds determined using the monotic and dichotic multiple auditory steady-state response technique in normal-hearing subjects," Scandinavian Audiology, vol. 30, no. 1, pp. 41-49, 2001.

[16] M. S. John, D. W. Purcell, A. Dimitrijevic, and T. W. Picton, "Advantages and caveats when recording steady-state responses to multiple simultaneous stimuli," Journal of the American Academy of Audiology, vol. 13, no. 5, pp. 246-259, 2002.

[17] T. W. Picton, M. S. John, A. Dimitrijevic, and D. Purcell, "Human auditory steady-state responses," International Journal of Audiology, vol. 42, no. 4, pp. 177-219, 2003.

[18] J. Hatton and D. R. Stapells, "The efficiency of the singleversus multiple-stimulus auditory steady state responses in infants," Ear and Hearing, vol. 32, no. 3, pp. 349-357, 2011.

[19] O. G. Lins and T. W. Picton, "Auditory steady-state responses to multiple simultaneous stimuli," Electroencephalography and Clinical Neurophysiology, vol. 96, no. 5, pp. 420-432, 1995.

[20] M. Armstrong and D. R. Stapells, "Multiple-stimulus interactions in the brainstem $(80 \mathrm{~Hz})$ and cortical $(14 \& 40 \mathrm{~Hz})$ auditory steady-state responses," in Proceedings of the 20th Biennial Symposium of the International Evoked Response Audiometry Study Group (IERASG), Bled, Slovenia, 2007.

[21] L. L. Wood, Multiple brainstem auditory steady-state response interactions for different stimuli [M.S. thesis], School of Audiology and Speech Sciences, The University of British Columbia, Vancouver, Canada, 2009.

[22] C. M. Fontaine and D. R. Stapells, "Efficiency of single versus multiple stimuli for $40-\mathrm{Hz}$ auditory steady-state responses," in Proceedings of the 20th Biennial Symposium of the International
Evoked Response Audiometry Study Group (IERASG), Bled, Slovenia, 2007.

[23] C. Petitot, L. Collet, and J. D. Durrant, "Auditory steadystate responses (ASSR): effects of modulation and carrier frequencies," International Journal of Audiology, vol. 44, no. 10, pp. 567-573, 2005.

[24] B. C. J. Moore, An Introduction to the Psychology of Hearing, Emerald Group Publishing, Bingley, UK, 6th edition, 2012.

[25] M. S. John and T. W. Picton, "MASTER: a windows program for recording multiple auditory steady-state responses," Computer Methods and Programs in Biomedicine, vol. 61, no. 2, pp. 125-150, 2000.

[26] S. A. Small and D. R. Stapells, "Normal ipsilateral/contralateral asymmetries in infant multiple auditory steady-state responses to air- and bone-conduction stimuli," Ear and Hearing, vol. 29, no. 2, pp. 185-198, 2008.

[27] S. A. Small and D. R. Stapells, "Artifactual responses when recording auditory steady-state responses," Ear and Hearing, vol. 25, no. 6, pp. 611-623, 2004.

[28] M. S. John, A. Dimitrijevic, and T. W. Picton, "Weighted averaging of steady-state responses," Clinical Neurophysiology, vol. 112, no. 3, pp. 555-562, 2001.

[29] L. Mo and D. R. Stapells, "The effect of brief-tone stimulus duration on the brain stem auditory steady-state response," Ear and Hearing, vol. 29, no. 1, pp. 121-133, 2008.

[30] B. Ross, R. Draganova, T. W. Picton, and C. Pantev, "Frequency specificity of $40-\mathrm{Hz}$ auditory steady-state responses," Hearing Research, vol. 186, no. 1-2, pp. 57-68, 2003.

[31] T. W. Picton, A. Dimitrijevic, M. C. Perez-Abalo, and P. van Roon, "Estimating audiometric thresholds using auditory steady-state responses," Journal of the American Academy of Audiology, vol. 16, no. 3, pp. 140-156, 2005.

[32] H. Thai-Van, C. Micheyl, A. Norena, E. Veuillet, D. Gabriel, and L. Collet, "Enhanced frequency discrimination in hearingimpaired individuals: a review of perceptual correlates of central neural plasticity induced by cochlear damage," Hearing Research, vol. 233, no. 1-2, pp. 14-22, 2007.

[33] E. C. Levi, R. C. Folsom, and R. A. Dobie, "Amplitudemodulation following response (AMFR): effects of modulation rate, carrier frequency, age, and state," Hearing Research, vol. 68 , no. 1 , pp. 42-52, 1993. 


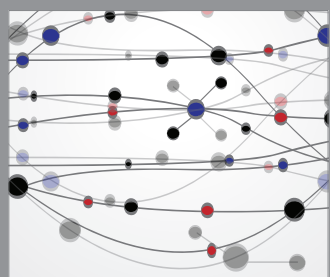

The Scientific World Journal
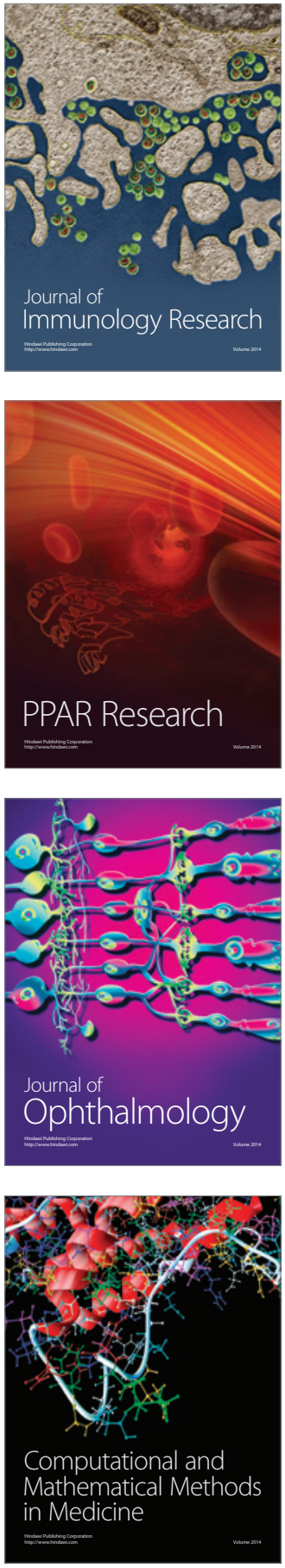

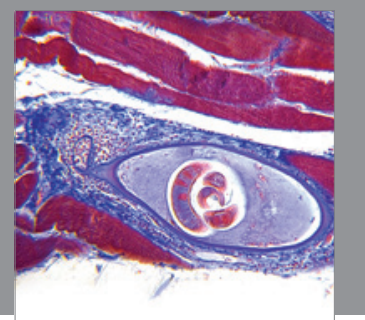

Gastroenterology

Research and Practice
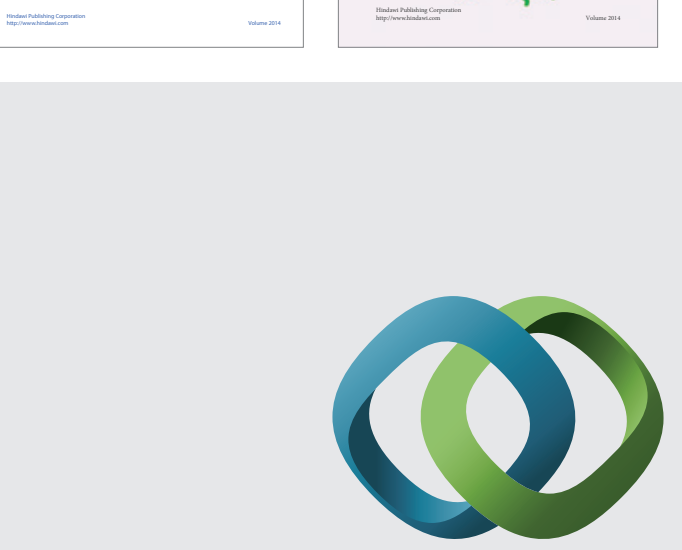

\section{Hindawi}

Submit your manuscripts at

http://www.hindawi.com
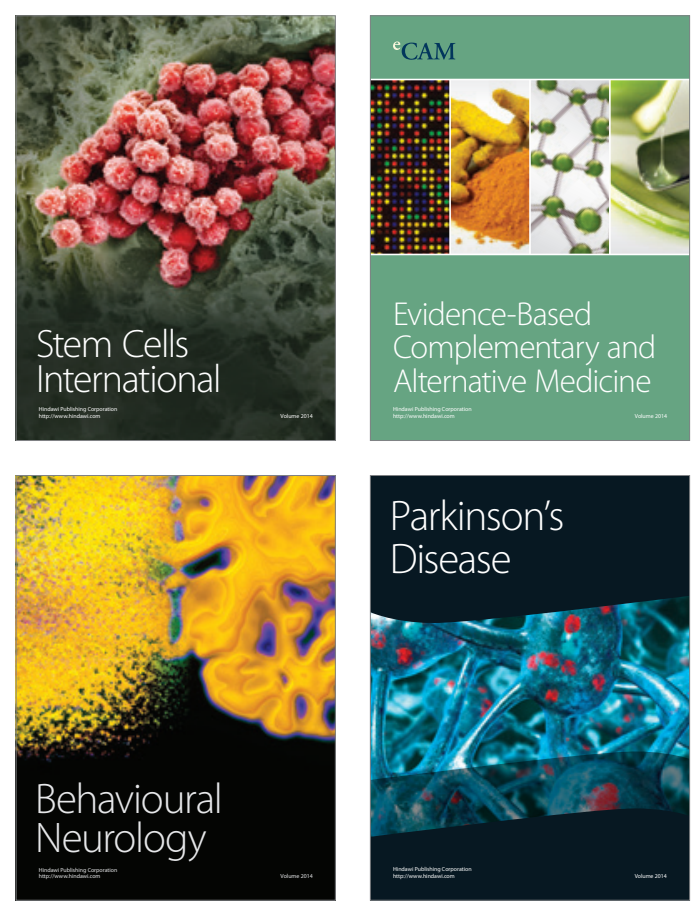

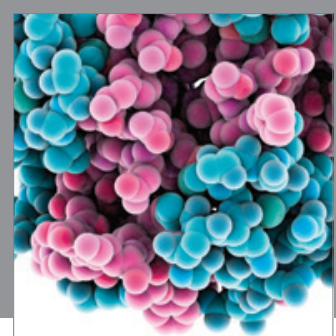

Journal of
Diabetes Research

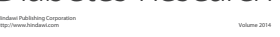

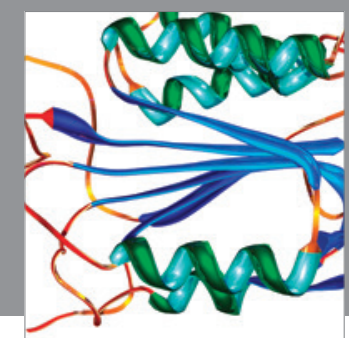

Disease Markers
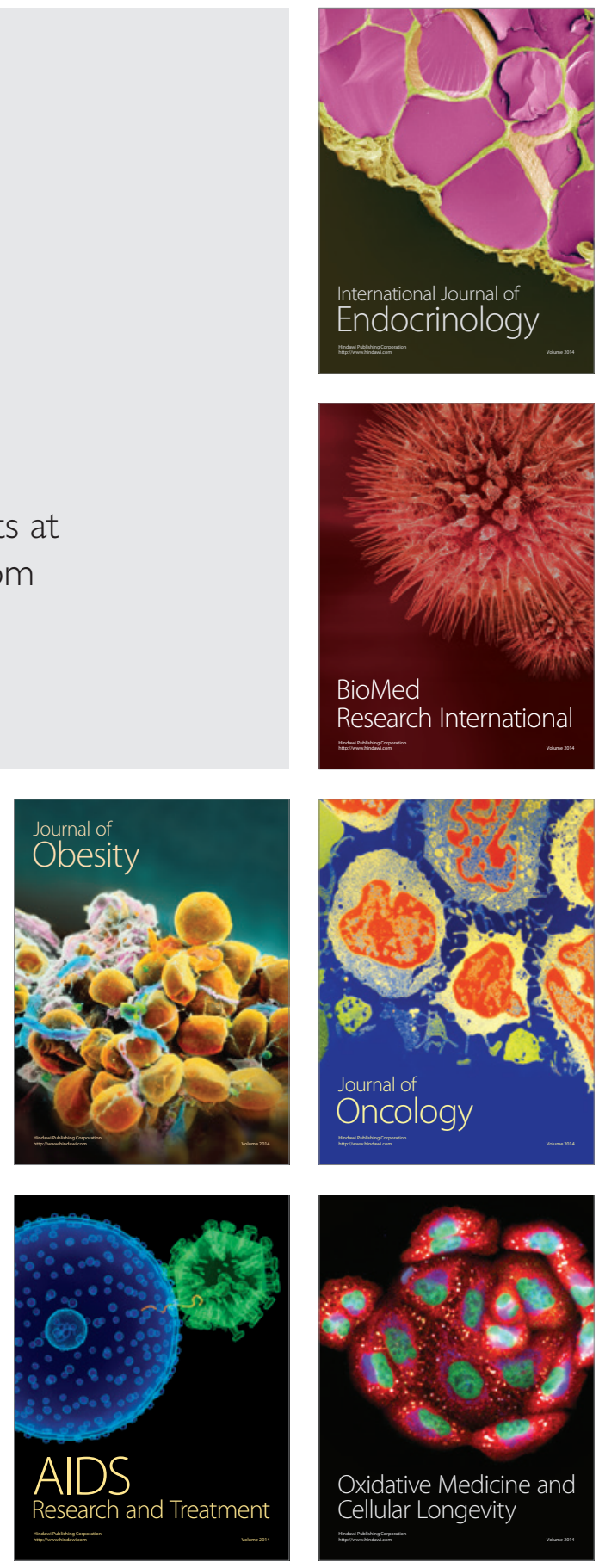